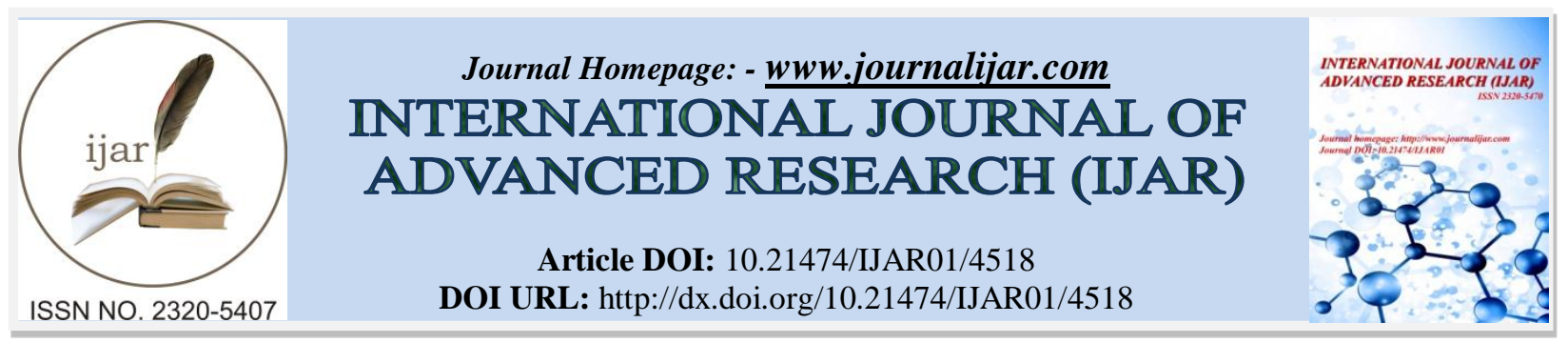

RESEARCH ARTICLE

\title{
INHIBITION OF MELANIN SYNTHESIS BY ASPERGILLUS FLAVUS SW-1 ISOLATED FROM SOIL.
}

Yeon Jo Ha, Sam Woong Kim, and "Sang Wan Gal.

Department of Pharmaceutical Engineering, Gyeongnam National University of Science and Technology.

\section{Manuscript Info}

Manuscript History

Received: 18 April 2017

Final Accepted: 20 May 2017

Published: June 2017

\section{Abstract}

The goal of this study is to find bacterial strains that inhibit production of melanin owing to suppression of tyrosinase and to find products that are less irritating skin than existing chemical products. We collected various soil samples from sea and mountain and examined the activity. Among them, we identified Aspergillus flavus SW-1 to suppress production of melanin. According to a result of tyrosinase activity in the supernatant of this strain, the inhibitor was suppressed depending on concentration of the supernatant. In addition, the activity was maintained very stable up to $100^{\circ} \mathrm{C}$. Taken together, since the supernatant of $A$. flavus SW-1 reduces biosynthesis of melanin, it is estimated to be highly applicable as a raw material for whitening functional cosmetics.

Copy Right, IJAR, 2017,. All rights reserved.

\section{Introduction:-}

A woman to a recent era have highly interested in whitening. A cosmetic to help skin brighten is called a whitening cosmetic product. In order to lighten skin, it is necessary to reduce melanin pigment which is a causative pigment to make skin dark. Melanin is a phenolic biopolymer substance widely distributed in nature and is a black pigment and protein complex. Melanin is biosynthesized by a melanocyte in pigment cell called melanocyte present in epidermal basal layer. Main production process of melanin is oxidized by tyrosinase from tyrosine, one of amino acids, to dioxyphenylalanine (DOPA), dopaquinone, and then produced to a melanin polymer through a chain reaction and final polymerization reaction (Hearing and Ekel, 1976; Nita and Young, 2005).

Melanin has been created to protect our skin that is damaged by ultraviolet rays (UV). As ultraviolet rays is a generic name of electromagnetic waves ranged in a wavelength of 10 to $397 \mathrm{~nm}$, when photographed by spectrum of sunlight, it means invisible light of a wavelength shorter than visible light. UV is also used for sterilizing and disinfecting equipment owing to a strong sterilizing function (KPSA, 1993). Properly exposure to sunlight is necessary for health of skin as well as sunlight promotes metabolism of skin to become strong skin owing to formation of appropriate melanin. This means that a melanin pigment is formed by a protective action on human body by ultraviolet rays. However, as exposure to sunlight for an excessively long time reduces low molecular weight antioxidants such as glutathione, vitamin E, vitamin $\mathrm{C}$ and ubiquinol, skin derives faster skin aging and rough due to excessive active oxygen, and becomes darker through formation of excessive melanin at the same time as wrinkles deepen, and deposits more melanin, which it can cause as a complex in skin beauty such as spots and freckles. Although a variety of raw materials have been developed, since some materials are excellent whitening effect in test tube, but they maintain side effects as well as the developed raw materials are high import dependence, high prices, and low competitiveness for enterprises in developed countries, whitening ingredients to be applied in 
cosmetics employ only a few types. Therefore, cosmetic companies are striving to develop natural or synthetic substances with whitening effect but have not succeeded easily. Currently, skin-whitening ingredients used in cosmetics are peeling agent to remove melanin pigment and synthetic inhibitor of skin melanin pigment (Maeda and Fukuda, 1991: Yamazaki and Kawano, 2010). As described above, synthetic inhibitor of melanin pigment prevents production of melanin by suppressing activity of tyrosinase, which is the most important factor for melanogenesis (Chang, 2009). However, since peeling agents for removing already produced melanin pigment shows peeling effect through breaking barrier function by separation between keratinocytes, they are not used due to side effects such as skin damage.

Therefore, in this study, we screened soil microorganisms to inhibit the activity of tyrosinase, but to prevent the formation of melanin while less irritating to skin than the existing chemical products. The screened microorganisms were studied for characteristics of the activity and effectiveness.

\section{Materials and Methods:-}

\section{Isolation and identification of melanin degrading strain:-}

Samples were applied by soils collected in East sea, South sea and nearby mountains in South Korea. Sample solutions were prepared by mixing $1 \mathrm{~g}$ of the collected samples and $9 \mathrm{ml}$ of sterilized water. Ink soup Czapek broth (Table 1) was used to select a melanin degrading strain. In order to separate a strain which maintains excellent activity, samples were cultured in ink soup Czapek broth, $100 \mathrm{ul}$ of cultural broth diluted to $10^{-3}$ was spread on LB agar plate and then single colony was induced on LB agar plate. The previous process was repeated by multiple times to purely isolate the selected strains. 16S rDNA sequence was done to identify pure-isolated strains (Macrogen, South Korea).

\section{Analysis of Tyrosinase inhibitory Effect:-}

To measure tyrosinase activity inhibition rate, after adding $25 \mathrm{ul}$ of $10 \mathrm{mM}$ L-tyrosine (Table. 2) to 96 well plate (SPL, Korea), a supernatant of sample was added for each concentration, 3 ul of mushroom tyrosinase (2,500 unit, Sigma Chemical Co., USA) was added, and the mixture was adjusted to a final 200 ul with $50 \mathrm{mM}$ phosphate buffer solution. The solution was reacted for $30 \mathrm{~min}$ at $30^{\circ} \mathrm{C}$, and absorbance was measured at $490 \mathrm{~nm}$ using an ELISA reader (Dynex, USA) at 1 min intervals during the reaction. The tyrosinase activity inhibition rate was determined by the following formula.

$$
\text { Tyrosinase activity inhibition rate (IC rate } \%)=100-\frac{\text { absorbance after reaction of sample solution }}{\text { absorbance after control solution }} \times 100
$$

\section{Analysis of thermal Stability of sample supernatant:-}

To analyze the thermal stability of the sample supernatant, the supernatant was reacted at 60 and $100^{\circ} \mathrm{C}$ for $30 \mathrm{~min}$, and then measurement of inhibitory activity of tyrosinase was carried out by the method described above.

Table. 1:- Melanin Czapek broth.

\begin{tabular}{|c|c|}
\hline \multicolumn{2}{|c|}{ Melanin Czapek broth } \\
\hline Melanin (0.2\%) & $2 \mathrm{~g} / \mathrm{L}$ \\
\hline $\mathbf{N H}_{\mathbf{4}} \mathbf{C l}$ & $3 \mathrm{~g} / \mathrm{L}$ \\
\hline $\mathbf{K}_{\mathbf{2}} \mathrm{HPO}_{4}$ & $1 \mathrm{~g} / \mathrm{L}$ \\
\hline $\mathbf{M g S O}_{4}$ & $0.5 \mathrm{~g} / \mathrm{L}$ \\
\hline $\mathrm{KCl}$ & $0.5 \mathrm{~g} / \mathrm{L}$ \\
\hline FeSO $_{4}$ & $0.01 \mathrm{~g} / \mathrm{L}$ \\
\hline Yeast extract & $1 \mathrm{~g} / \mathrm{L}$ \\
\hline
\end{tabular}


Table. 2:- Tyrosine composition applied for melanin synthesis

\begin{tabular}{|c|c|}
\hline \multicolumn{2}{|c|}{ L-Tyrosine } \\
\hline Assay & above $99.00 \%$ \\
\hline Drying loss & below $0.10 \%$ \\
\hline Ignition on Residue & below $0.05 \%$ \\
\hline Chloride(CI) $(\mathbf{C l})$ & below $0.02 \%$ \\
\hline Sulfate(SO $)$ & below $0.02 \%$ \\
\hline Iron(Fe) & below $10 \mathrm{ppm}$ \\
\hline Heavy metals (As and $\mathbf{P b})$ & below $10 \mathrm{ppm}$ \\
\hline
\end{tabular}

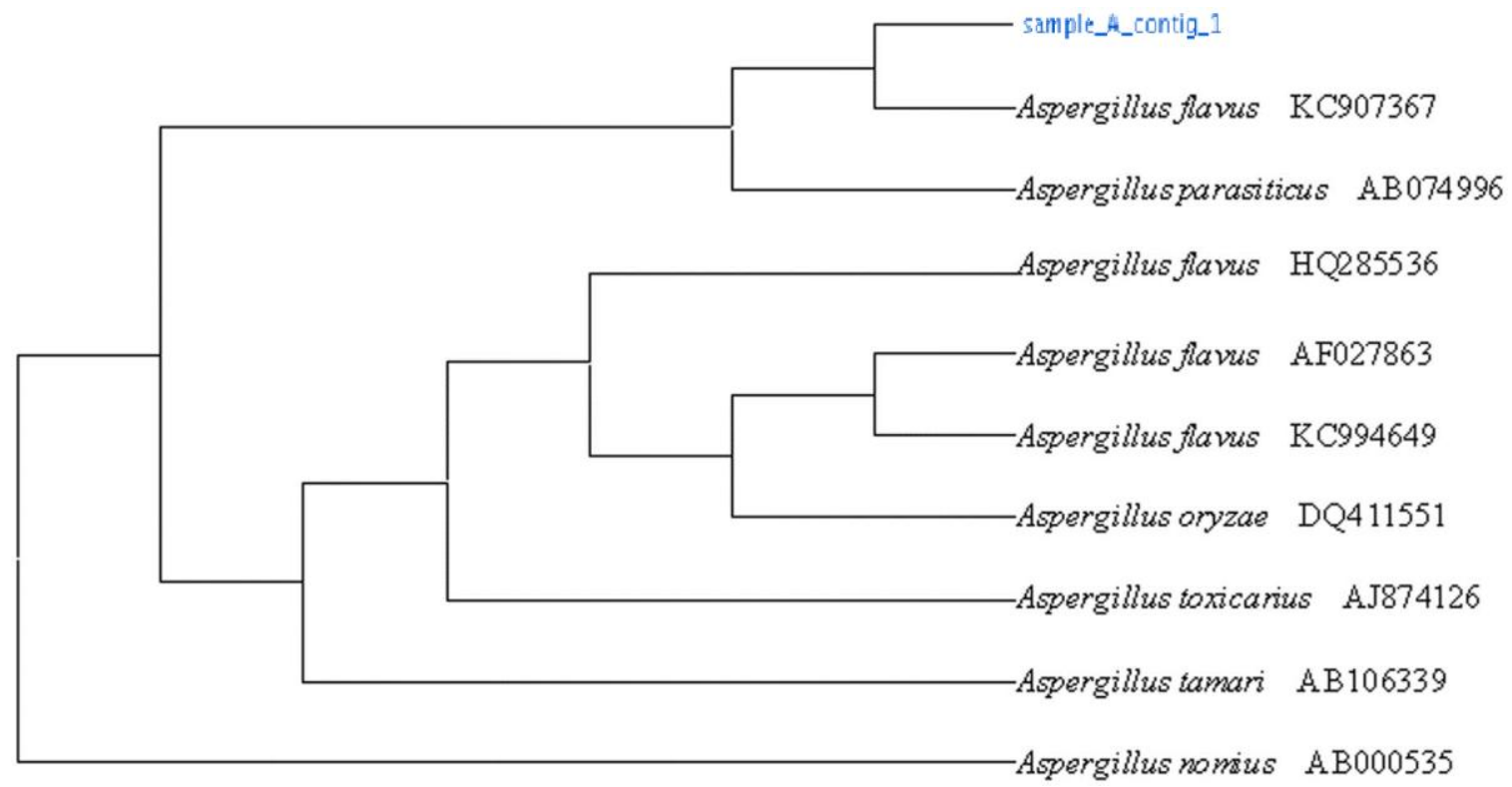

Fig. 1:- Phylogenetic classification of the isolated sample.
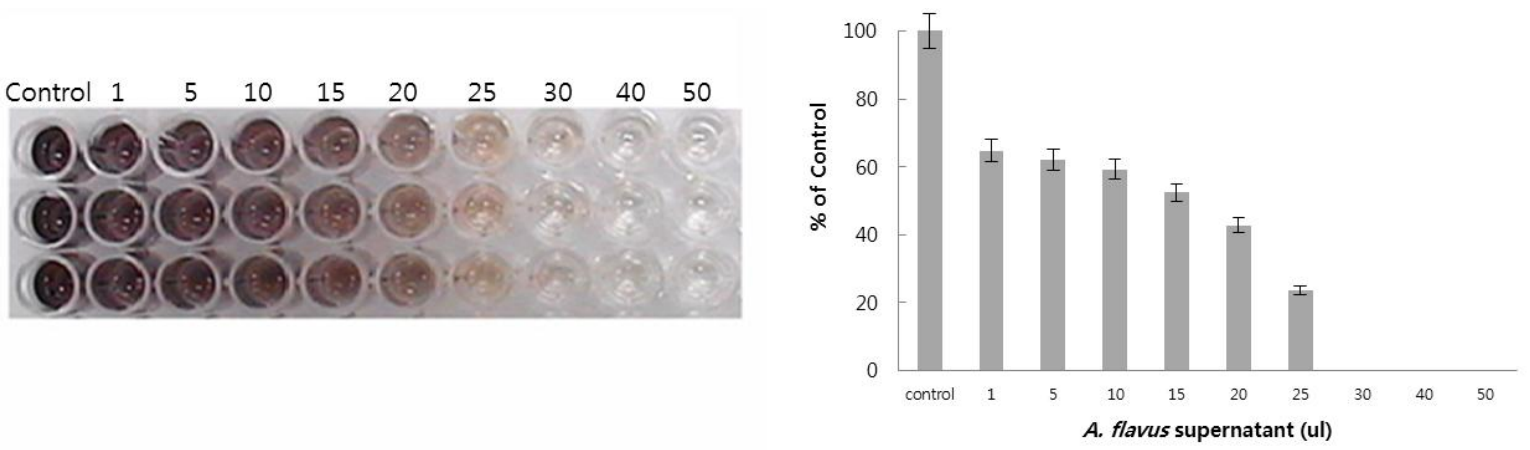

Fig. 2:- Effect of inhibitor(s) to tyrosinase by $A$. flavus SW-1 supernatant. The measurement of tyrosinase activity was done by reaction mixture including L-tyrosine, A. flavus $S W$-1supernatant, mushroom tyrosinase, phosphate buffer. The reaction mixture was treated for $30 \mathrm{~min}$ at $30^{\circ} \mathrm{C}$ and then measured by $490 \mathrm{~nm}$ in wavelength with ELISA reader (Dynex, USA). X- and Y-axes of bar graph indicate the employed extract concentration and relative activity of tyrosinase, respectively. 

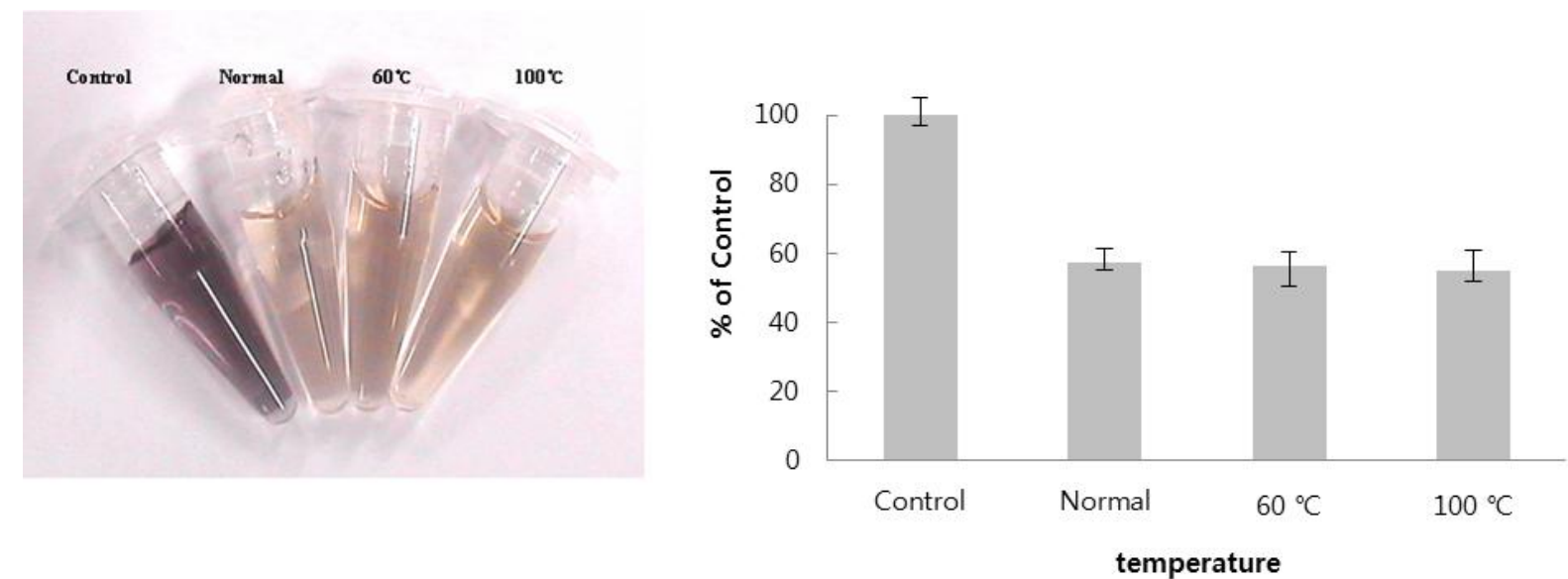

Fig. 3:- Evaluation of heat stability to tyrosinase activity by $A$. flavus $\mathrm{SW}-1$ supernatant. The studied method was applied by same procedure with Fig. 2, except for pre-treatment by heat to the employed extracts, which were done for $30 \mathrm{~min}$ at 60 and $100^{\circ} \mathrm{C}$. X-and $\mathrm{Y}$-axes of bar graph indicate the employed extract concentration and relative activity of tyrosinase, respectively.

\section{Results and Discussion:-}

Strain identification and evaluation of tyrosinase inhibition activity from A. flavus SW-1 supernatant:-

A strain capable of inhibiting tyrosinase activity was isolated from nature via screening, and then the isolated strain with the activity was identified by $16 \mathrm{~s}$ rDNA sequence. As a result, since the strain showed high homology with Aspergillus flavus $\mathrm{SW}-1$, the isolated strain was named as A. flavus SW-1(Fig. 1).

To examine whether inhibition of tyrosinase suppresses melanin synthesis to some extent, the tyrosinase inhibitory activity was measured by $10 \mathrm{mM}$ L-tyrosine, 3 ul of mushroom tyrosinase (2,500 unit, Sigma Chemical Co., USA) and each concentration of A. flavus SW-1 supernatant. For a control, a $50 \mathrm{mM}$ phosphate buffer solution was used in place of the A. flavus SW-1 supernatant. As a result, the inhibition of tyrosinase activity was found to be carried out depending on the concentration of the supernatant (Fig. 2). In previous study, cultural broth of Aspergillus fumigatus induces degradation of melanin (Luther and Lipke, 1980). In addition, it is found that tyrosinase-related protein-1 (TRP-1), TRP-2, and microphthalmia-associated transcription factor (MITF) of medicinal mushroom Ganoderma lucidum suppress melanin biosynthesis (Kim et al., 2016). In this study, it is assumed that A. flavus SW-1 supernatant is capable of suppressing melanin biosynthesis via tyrosinase inhibition.

\section{Evaluation of thermal stability for $A$. flavus SW-1 supernatant:-}

In order to investigate whether tyrosinase inhibitory component of A. flavus SW-1 supernatant is safe against temperature, the supernatant was reacted for $30 \mathrm{~min}$ at 60 and $100^{\circ} \mathrm{C}$, and then examined for the inhibitory effect of tyrosinase. As shown in Fig. 3, it is assumed that the activity is very stable at temperature owing to the same results as the untreated group. Tyrosinase-inhibitory activity of benzyldithiocarbamate decreases activity depending on temperature, whereas p-xylidine-bis dithiocarbamate shows a pattern of increase (Amin et al., 2010). Therefore, it is assumed that tyrosinase inhibitors play roles by substances with various chemical properties.

\section{Conclusion:-}

Although Aspergillus flavus is a widely distributed fungus in normal soil and already a lot of studies appeared, data for suppressing melanin synthesis have been showed a little studies. Therefore, we used a supernatant obtained from A. flavus SW-1 which proceeds research to suppress synthesis of melanin. Tyrosinase-inhibitory effect was suppressed in proportion to the concentration of A. flavus SW-1 supernatant. Evaluation of thermal stability showed the same result as control group without heat treatment. Therefore, we suggest that A. flavus SW-1 supernatant has tyrosinase inhibitory effect and excellent thermal stability.

\section{Acknowledgements:-}

This work was supported by Gyeongnam National University of Science and Technology Grant 2015. 


\section{Reference:-}

1. Amin E, Saboury AA, Mansuri-Torshizi H, Moosavi-Movahedi AA. 2010. Potent inhibitory effects of benzyl and p-xylidine-bis dithiocarbamate sodium salts on activities of mushroom tyrosinase. J Enzyme Inhib Med Chem. 25(2), 272-281.

2. Chang TS. 2009. An updated review of tyrosinase inhibitors. International Journal of Molecular Sciences, 10, 2440-2475.

3. Hearing VJ. and Ekel T. M. 1976. Mammalian tyrosinase. Biochem. J. 157, 549-557.

4. Kim JW, Kim HI, Kim JH, Kwon OC, Son ES, Lee CS, Park YJ. 2016. Effects of ganodermanondiol, a new melanogenesis inhibitor from the medicinal mushroom Ganoderma lucidum. Int J Mol Sci. 17(11), pii: E1798.

5. Korean Products Safety Association (KPSA). 1993. "Quality comparison evaluation - Electro-disinfector (Ultraviolet)" Electricity and safety 12, 39-51.

6. Luther JP and Lipke H. 1980. Degradation of melanin by Aspergillus fumigatus. Applied and Environmental Microbiology, 40, 145 155.

7. Maeda K. and Fukuda M. 1991. In vitro effectiveness of several whitening cosmetic components in human melanocytes. J. Soc. Cosmet. Chem. 42, 361-368.

8. Nita A. and Young AR. 2005. Melanogenesis: a photoprotective response to DNA damage? Mutation Research, 571, 121-132.

9. Yamazaki Y. and Kawano Y. 2010. N-(3,5-dihydroxybenzoyl)-6 -hydroxytryptamine as a novel human tyrosinase inhibitor that inactivates the enzyme in cooperation with L-3,4-dihydroxyphenylalanine. Chem. Pharm. Bull, 58, 1536-1540. 